\title{
Video Article \\ Use of Anti-phospho-girdin Antibodies to Visualize Intestinal Tuft Cells in Free-Floating Mouse Jejunum Cryosections
}

\author{
Yuka Mizutani ${ }^{1}$, Daisuke Kuga ${ }^{2}$, Machiko lida ${ }^{1}$, Kaori Ushida ${ }^{3}$, Tsuyoshi Takagi ${ }^{1}$, Yoshihito Tokita ${ }^{1}$, Masahide Takahashi ${ }^{3}$, Masato Asai ${ }^{1,3}$ \\ ${ }^{1}$ Division of Perinatology, Institute for Developmental Research, Aichi Human Service Center \\ ${ }^{2}$ Surgery Department, Anjo Kosei Hospital \\ ${ }^{3}$ Department of Pathology, Nagoya University Graduate School of Medicine
}

Correspondence to: Masato Asai at masato-a@inst-hsc.jp

URL: https://www.jove.com/video/57475

DOI: doi: $10.3791 / 57475$

Keywords: Biology, Issue 133, Tuft Cells, Antibodies against Girdin Phosphorylated at Tyrosine 1798, Phalloidin, Gelatin-Filled Intestine, FreeFloating Cryosection, Low-Temperature Antigen Retrieval

Date Published: $3 / 21 / 2018$

Citation: Mizutani, Y., Kuga, D., lida, M., Ushida, K., Takagi, T., Tokita, Y., Takahashi, M., Asai, M. Use of Anti-phospho-girdin Antibodies to Visualize Intestinal Tuft Cells in Free-Floating Mouse Jejunum Cryosections. J. Vis. Exp. (133), e57475, doi:10.3791/57475 (2018).

\section{Abstract}

The actin binding protein girdin is a cytosolic protein that is required for actin remodeling to trigger cell migration in various tissues. Girdin is phosphorylated by both receptor and non-receptor tyrosine kinases at tyrosine 1798. Omori et al. developed site- and phosphorylation status-specific antibodies against human girdin at tyrosine-1798 (pY1798), which specifically bind to phosphorylated tyrosine-1798, but not to unphosphorylated tyrosine-1798. pY1798 antibodies have been used to specifically label tuft cells (TCs) that are present in mammalian gastrointestinal tissues, but the function of these cells is unclear. This protocol allows the robust visualization of TCs in the jejunum using pY1798 antibodies and immunofluorescence. To ensure successful and simple TC visualization, this protocol includes two histological techniques: production of free-floating cryosections from gelatin-filled jejunum tissue, and low-temperature antigen retrieval at $50{ }^{\circ} \mathrm{C}$ for $3 \mathrm{~h}$. Filling the jejunum with gelatin maintains the shape of free-floating sections throughout the staining procedure, whereas low-temperature antigen retrieval ensures robust signals from TCs. Successful use of this protocol results in pY1798 staining of TCs distributed from villus tip to crypt. Stained TCs have a spool-shaped soma and fluorescent signals condense at the lumenal tip, which corresponds to the protruding 'tuft.' Phalloidin staining colocalized with pY1798-positive TCs at the thickened brush border, and corresponds to a rootlet mass extending from the TC tuft. This protocol could be used to examine TCs in human biopsy samples collected with gastrointestinal endoscopes. Furthermore, TCs were recently reported to accumulate following parasite infection in mice, suggesting that this protocol could have applications for diagnosis of parasite infections in the human gut.

\section{Video Link}

The video component of this article can be found at https://www.jove.com/video/57475/

\section{Introduction}

Tuft cells (TCs) are minor scattered components of gastrointestinal epithelia that are characterized by apical tufts and spool-shaped somas ${ }^{1}$ Although TCs were first described in $1956^{2}$, TC function remains unclear, partly due to a lack of reliable TC markers.

Enomoto et al. first characterized the actin binding protein girdin ${ }^{3}$, which is expressed in the nervous system, as well as in non-neural tissues such as blood vessels, heart valves, tendons, and skeletal muscle ${ }^{4}$. Mice with genetic ablation of girdin display growth retardation and multiple brain anomalies ${ }^{4,5,6}$. Meanwhile, loss-of-function mutation in human girdin is associated with progressive encephalopathy, severe retardation, and early onset epileptic seizures?

In 2011, Lin et al. identified dynamic phosphorylation of girdin at tyrosine 1798 mediated by tyrosine kinases such as EGFR and Src ${ }^{8}$. They also showed that phosphorylated girdin is required for actin remodeling to trigger cell migration ${ }^{8}$. Omori et al. developed site- and phosphorylation status-specific antibodies against human girdin phosphorylated at tyrosine 1798 (pY1798 antibodies) following Goto's protocol, and validated the antibodies using site-directed mutagenesis of expression vectors carrying full-length girdin ${ }^{9,10}$. In 2017 , Kuga et al. reported that pY1798 labels TCs with high specificity and high sensitivity ${ }^{1}$. The pY1798 antibodies were shown to be superior to previous TC markers based on excellent staining properties that revealed the whole cell shape, including the characteristic 'tuft' on the lumenal tip, yet the biological role of girdin and phospho-girdin in TC function remained unclear ${ }^{1}$.

The method described in this study involves immunofluorescence staining, a histological technique to mark a specific protein on tissue using a primary antibody against a target protein and a fluorescence-conjugated secondary antibody against the primary antibody. The purpose of this method is to allow researchers with limited histological experience to obtain high quality TC images. This protocol uses free-floating cryosections that avoid the need for slide-mounted cryosections, or paraffin section. However, free-floating sections are fragile due to the absence of support from a glass slide and the section thickness can lessen antibody permeability. This protocol includes two approaches that overcome these 
issues: 1) filling the entire jejunum lumen with gelatin to preserve the morphology of free-floating sections throughout the staining procedures, and 2) use of low-temperature antigen retrieval to intensify pY1798 signals.

Protocol

All methods described here were approved by the Animal Care and Use Committee of the Institute for Developmental Research, Aichi Human Service Center (application number: M-03).

\section{Preparations}

1. Prepare $10 \mathrm{~L}$ of phosphate buffered saline (PBS): $32.27 \mathrm{~g}$ of $\mathrm{Na}_{2} \mathrm{HPO}_{4} \cdot 12 \mathrm{H}_{2} \mathrm{O}, 4.5 \mathrm{~g}$ of $\mathrm{NaH}_{2} \mathrm{PO}_{4} \cdot 2 \mathrm{H}_{2} \mathrm{O}, 80.0 \mathrm{~g}$ of $\mathrm{NaCl}$ added to ultrapure water to a final volume of $10 \mathrm{~L}$. Store solution at room temperature (RT).

2. Prepare $200 \mathrm{~mL}$ of PBS-T: $200 \mathrm{~mL}$ of PBS from step 1.1 with $100 \mu \mathrm{L}$ of polyoxyethylene(10) octylphenyl ether to a final concentration of $0.05 \%$ (vol:vol). Store at RT.

3. While wearing gloves and eye protection, prepare $50 \mathrm{~mL}$ of $15 \%$ sucrose in $10 \%$ buffered neutral formalin solution: $7.5 \mathrm{~g}$ of sucrose added to $10 \%$ buffered neutral formalin solution (Table of Materials) to a final volume of $50 \mathrm{~mL}$. Store at RT.

CAUTION: Inhalation and/or skin/eye contact with formaldehyde in $10 \%$ buffered neutral formalin solution can be hazardous. Handle with caution.

4. Prepare $1.5 \mathrm{~mL}$ of 200 units $/ \mathrm{mL}$ phalloidin-fluorescent dye conjugate stock solution: phalloidin-fluorescent dye conjugate (300 units in 1 vial, lyophilized solids, excitation and emission wavelengths: $581 \mathrm{~nm}$ and $609 \mathrm{~nm}$, respectively) suspended in $1.5 \mathrm{~mL}$ of methanol. Store solution in the dark at $-20^{\circ} \mathrm{C}$.

5. Prepare $5 \mathrm{mg} / \mathrm{mL}$ 4',6-diamidino-2-phenylindole, dihydrochloride (DAPI) stock solution: $10 \mathrm{mg}$ of DAPI in $2 \mathrm{~mL}$ of N,N-dimethylformamide (DMF). Store solution in the dark at $-20^{\circ} \mathrm{C}$

6. Prepare $5 \%$ bovine serum albumin (BSA) in PBS: $0.5 \mathrm{~g}$ of BSA, $10 \mathrm{~mL}$ of PBS. Store at $4{ }^{\circ} \mathrm{C}$.

7. Remove the beveled tip of an 18-gauge straight needle using a nipper, and pinch the nipped end with a pincher in a lengthwise direction to allow liquid to flow through the needle lumen (Figure 1A1). NOTE: The protocol can be paused here.

\section{Animal Dissection and Isolation of Jejunum}

1. Perfusion fixation of a mouse

1. Wear gloves and work in a well-ventilated area.

2. Prepare a $100 \mathrm{~mL}$ glass beaker, $10 \%$ buffered neutral formalin solution, surgical tools (scissors, forceps), a metallic tray, 6-0 nylon cut sutures, an 18-gauge straight needle prepared as in 1.7, a 22-gauge winged needle, a $20-\mathrm{mL}$ syringe.

3. Load $20 \mathrm{~mL}$ of $10 \%$ buffered neutral formalin solution from the glass beaker into the $20-\mathrm{mL}$ syringe, and attach the $22-$ gauge winged needle to the outlet.

4. Prepare liquid gelatin by adding $1 \mathrm{~g}$ of gelatin powder to $20 \mathrm{~mL}$ of PBS (final $5 \%$ gelatin) in a $50-\mathrm{mL}$ centrifuge tube. After soaking at $\mathrm{RT}$ for $15 \mathrm{~min}$, incubate at $50^{\circ} \mathrm{C}$ in a water bath for 15 min without shaking.

5. Briefly vortex, and incubate at $50^{\circ} \mathrm{C}$ for another $15 \mathrm{~min}$ to completely dissolve the gelatin. Leave the tube at RT until use.

6. Euthanize an adult mouse by cervical dislocation.

7. Place the mouse from step 2.1.6 on a metallic tray and make a small incision on the skin through the ensiform cartilage using surgical tools.

8. Expand the incision with both hands to expose the thoracic and abdominal regions.

9. Open the peritoneum to expose the diaphragm, and then open the diaphragm on both sides.

10. Cut the thoracic cage along the anterior axillary lines on both sides, then remove the thoracic wall by cutting in a transverse direction at the position of thymus to expose the heart.

11. Make a small incision on the auricle of the right atrium to drain the blood and insert a 22-gauge winged needle into the apex of the left ventricle. Push the plunger to perfuse the tissues with $10 \%$ buffered neutral formalin solution.

12. After exposing organs in the pelvis, cut off the end of the rectum from the anus, and separate the intestines from the body by cutting the mesentery.

13. To isolate the jejunum, cut the intestines at $4 \mathrm{~cm}$ from the anal side of the gastric antrum. Discard the anal half of the remaining small intestine.

14. Clip the jejunum in half to facilitate the flushing procedure. Load $20 \mathrm{~mL}$ of $10 \%$ buffered neutral formalin solution into the 20 -mL syringe, and attach the 18-gauge straight needle prepared in step 1.7 to the outlet.

15. Inject $10 \%$ buffered neutral formalin solution from one end of the clipped jejunum to flush out the intestinal contents and to fix the gut lumen surface (Figure 1A2).

16. Wash the gut lumen by injecting PBS as described in step 2.1.15.

17. Flush liquid gelatin into the gut lumen as described in step 2.1.15 to replace PBS with liquid gelatin.

18. Close one end of the clipped jejunum by suture ligation using a 6-0 nylon cut suture, fill the jejunum with liquid gelatin, and close the opposite end by suture ligation (Figure 1A3).

19. Add four suture knots to fit a sausage-shaped jejunum section to the depressed portion of a cryomold (Figure 1A4). NOTE: For cryomolds with a $20 \mathrm{~mm} \times 25 \mathrm{~mm} \times 5 \mathrm{~mm}$ depressed section, a $\sim 20 \mathrm{~mm}$ sausage-like jejunum section is preferable.

20. Soak the tissues in $50 \mathrm{~mL}$ of $15 \%$ sucrose in $10 \%$ buffered neutral formalin solution overnight at $4{ }^{\circ} \mathrm{C}$. NOTE: These steps ensure cryoprotection by sucrose and protein fixation by formalin of gelatin and tissue. Formalin-gelatinized gelatin does not melt at RT, whereas non-fixed gelatinized gelatin at $4{ }^{\circ} \mathrm{C}$ melts at RT. 


\section{Snap Freezing of Gelatin-filled Jejunum Tissues}

1. By cutting the jejunum at the suture knots, three sausage-like jejunum pieces with both ends ligated are obtained (Figure 1A4). Align the sausage-like pieces in a cryomold for addition of embedding compound for preparation of frozen tissue specimens.

2. Snap freeze the cryomold in isopentane cooled with liquid nitrogen. Store cryomolds at $-80^{\circ} \mathrm{C}$ NOTE: The protocol can be paused here.

\section{Immunofluorescence of Tuft Cells Using Free-floating Cryosections}

1. Cryosectioning

1. Set both the cryostat chamber temperature (CT) and object temperature (OT) to $-22{ }^{\circ} \mathrm{C}$. Place the frozen tissue block in a cryomold in the cryostat chamber for at least $15 \mathrm{~min}$.

2. Add $3 \mathrm{~mL}$ of PBS to a 35-mm culture dish.

3. Remove the frozen tissue block from the cryomold and cut the block in half with a razor blade to expose the transverse section of the jejunum. Mount one half of the block on a chuck (a cryostat adaptor) to section the cutting plane with the razor blade.

4. Section the gelatin-filled jejunum into $30 \mu \mathrm{m}$-thick sections. Use frozen forceps to gently transfer the sections into the $35-\mathrm{mm}$ culture dish prepared in step 4.1.2 (Figure 2B, right).

2. Application of primary antibodies

1. Wash the free-floating sections in the $35-\mathrm{mm}$ culture dish 3 times for 5 min each with $3 \mathrm{~mL}$ PBS-T with mild shaking on a reciprocal shaker (approximately 36 times/min).

2. Prepare antigen retrieval solution: $0.3 \mathrm{~mL}$ of antigen retrieval solution concentrate (Table of Materials) in $2.7 \mathrm{~mL}$ of ultrapure water Add $3 \mathrm{~mL}$ of antigen retrieval solution to the $35-\mathrm{mm}$ culture dish containing free-floating sections.

3. Close the lid, seal the gap between the dish and the lid with a strip of vinyl tape, and incubate at $50{ }^{\circ} \mathrm{C}$ in a hybridization incubator for 3 $\mathrm{h}$ without shaking.

4. Remove the dish from the incubator and cool at RT for $20 \mathrm{~min}$. After removing the vinyl tape, wash the sections 3 times for 5 min each with $3 \mathrm{~mL}$ of PBS-T and mild shaking.

5. Aspirate the PBS-T and add 5 drops of blocking solution (Table of Materials) onto the sections. Incubate at RT for 5 min with mild shaking.

6. Prepare the primary antibody solution: $495 \mu \mathrm{L}$ of $5 \%$ BSA in PBS with $5 \mu \mathrm{L}$ of phospho-Y1798 girdin (pY1798) antibodies (Table of Materials). Add $500 \mu \mathrm{L}$ of primary antibody solution onto the sections (the blocking solution does not need to be removed).

7. Place the dish in a humidified incubation chamber and incubate overnight at $4{ }^{\circ} \mathrm{C}$ with mild shaking. NOTE: The overnight incubation can be extended to up to three nights.

3. Application of secondary antibodies

1. Wash the sections 3 times for 5 min each in $3 \mathrm{~mL}$ of PBS-T with mild shaking.

2. Prepare diluted DAPI stock solution: $2 \mu \mathrm{L}$ of DAPI stock solution (Step 1.5), $998 \mu \mathrm{L}$ of PBS.

3. Make secondary antibody solution: $476 \mu \mathrm{L}$ of $5 \%$ BSA in PBS, $10.5 \mu \mathrm{L}$ of diluted DAPI solution, $12.5 \mu \mathrm{L}$ of phalloidin-fluorescent dye conjugate stock solution, $1 \mu \mathrm{L}$ of goat anti-rabbit IgG-fluorescent dye conjugate (wavelength: excitation $496 \mathrm{~nm}$, emission $520 \mathrm{~nm}$ )

4. After aspirating the PBS-T, apply the secondary antibody solution, and incubate in a light-shielded incubation chamber at RT for 30 min with mild shaking.

5. Wash the sections 3 times for 5 min each with $3 \mathrm{~mL}$ of PBS-T and mild shaking

6. After the final wash, remove the PBS-T and replace with $3 \mathrm{~mL}$ of PBS lacking polyoxyethylene(10) octylphenyl ether. Transfer the dish to a stereoscopic microscope.

7. Place $200 \mu \mathrm{L}$ of PBS in a droplet on the center of a MAS-coated white glass slide and transfer one jejunum section from the dish into the droplet using a P200 pipet tip.

8. After adjusting the section alignment under the stereoscopic microscope, aspirate all remaining PBS surrounding the section.

9. Add $20 \mu \mathrm{L}$ of aqueous mounting media and place a $20 \times 20 \mathrm{~mm}$ coverslip atop the media.

10. Immediately seal the coverslip edges with xylene-based mounting media. Place the slide on a wooden mappe and allow the xylenebased mounting media to solidify at RT for $2-3 \mathrm{~h}$.

NOTE: The protocol can be paused here. After the xylene-based mounting media solidifies, the slides can be stored for 2 - 3 weeks in a light-shielded slide box at RT.

\section{Confocal Microscopy}

1. Put immersion oil on the $63 \mathrm{X}$ objective of a confocal microscope. Turn the coverslip-slide down and place the slide on the stage.

2. Digitize the TC images acquired at laser wavelengths 405,488 , and $555 \mathrm{~nm}$, and save the images in tiff format (.tiff). NOTE: Choose a detection filter set that is appropriate for the fluorescence dyes (i.e., excitation/emission maxima: $358 / 461 \mathrm{~nm}, 490 / 525$, and 590/617).

\section{Representative Results}

\section{Gelatin-filling of the jejunum}

A typical procedure for filling mouse jejunum with gelatin is shown (Figure 1A), as are the benefits of gelatin-filling (Figure 1B). In brief, the beveled tip of an 18-gauge straight needle was removed to protect against piercing the gut wall (Figure 1A1). Gelatin filling was achieved using $10 \%$ buffered neutral formalin solution injected from one end of a clipped jejunum section to flush the intestine and to fix the gut lumen 
surface (Figure 1A2) prior to filling with liquid gelatin (Figure 1A3). The resulting sausage-like pieces (Figure 1A4) were embedded, frozen, and transversely sectioned into $30 \mu \mathrm{m}$-thick slices in a cryostat. In the absence of gelatin filling, jejunal sections tend to kink, allowing the villi to swing backward (Figure 1B, left). Gelatin filling preserves the round disc-shape of the sections and maintains upright positioning of the villi (Figure 1B, right). The images clearly indicate the benefit afforded by gelatin filling in preserving the morphology of free-floating jejunum sections.

\section{Successful imaging of intestinal tuft cells}

pY1798 antibodies can be applied for variable immunostaining techniques, including dot blotting, western blotting, paraffin section staining, thawmounted cryosection staining, or free-floating cryosection staining ${ }^{1,9}$. In this protocol, we focused on free-floating cryosections for fluorescence staining of TCs in mouse jejunum using pY1798 antibodies, phalloidin, and DAPI staining to demonstrate the structural characteristics of TCs ${ }^{1}$. In general, TCs are scattered at a rate of approximately one TC/100 epithelial cells from villus tip to crypt ${ }^{1}$. The representative results show that pY1798 reproducibly delineates entire TCs, including the membrane, cytoplasm of the spool-shaped soma, and the strongly stained lumenal tip, where robust signal condensation corresponds to the protruding 'tuft' of a TC ${ }^{1}$ (Figure 2A-2B). Meanwhile, phalloidin is a phallotoxin, a family of poisonous bicyclic heptapeptides from the mushroom Amanita phalloides, and has high affinity for filamentous actin ( $F$-actin), which is present in microvilli that form the intestinal brush border ${ }^{11}$. Phalloidin reproducibly and prominently marks the thickened brush border that corresponds to a mass of rootlets extending from the tuft ${ }^{1}$. Thus, the consistent co-localization of pY1798 signals (spool-shaped soma, signal condensation at the lumenal tip) with the prominently thickened phalloidin-positive brush border demonstrates that this protocol successfully identifies TCs regardless of whether they are located on a villus (Figure 2A) or in a crypt (Figure 2B).

\section{Low-temperature antigen retrieval is effective for staining free-floating sections}

Heat-based antigen retrieval at $95-99^{\circ} \mathrm{C}$ is widely used for analysis of paraffin sections and slide-mounted cryosections. However, applying this approach to free-floating sections without causing damage is difficult because such sections are generally more fragile than slidemounted sections that are supported by the slide ${ }^{12}$. Since low-temperature antigen retrieval $\left(50^{\circ} \mathrm{C}\right.$ for $3 \mathrm{~h}$ ) using a water bath did not affect the morphology of the free-floating jejunum sections in preliminary experiments, we evaluated the objective effectiveness of antigen retrieval in a blind test, which statistically confirmed the effectiveness of low-temperature antigen retrieval (Figure 3). 

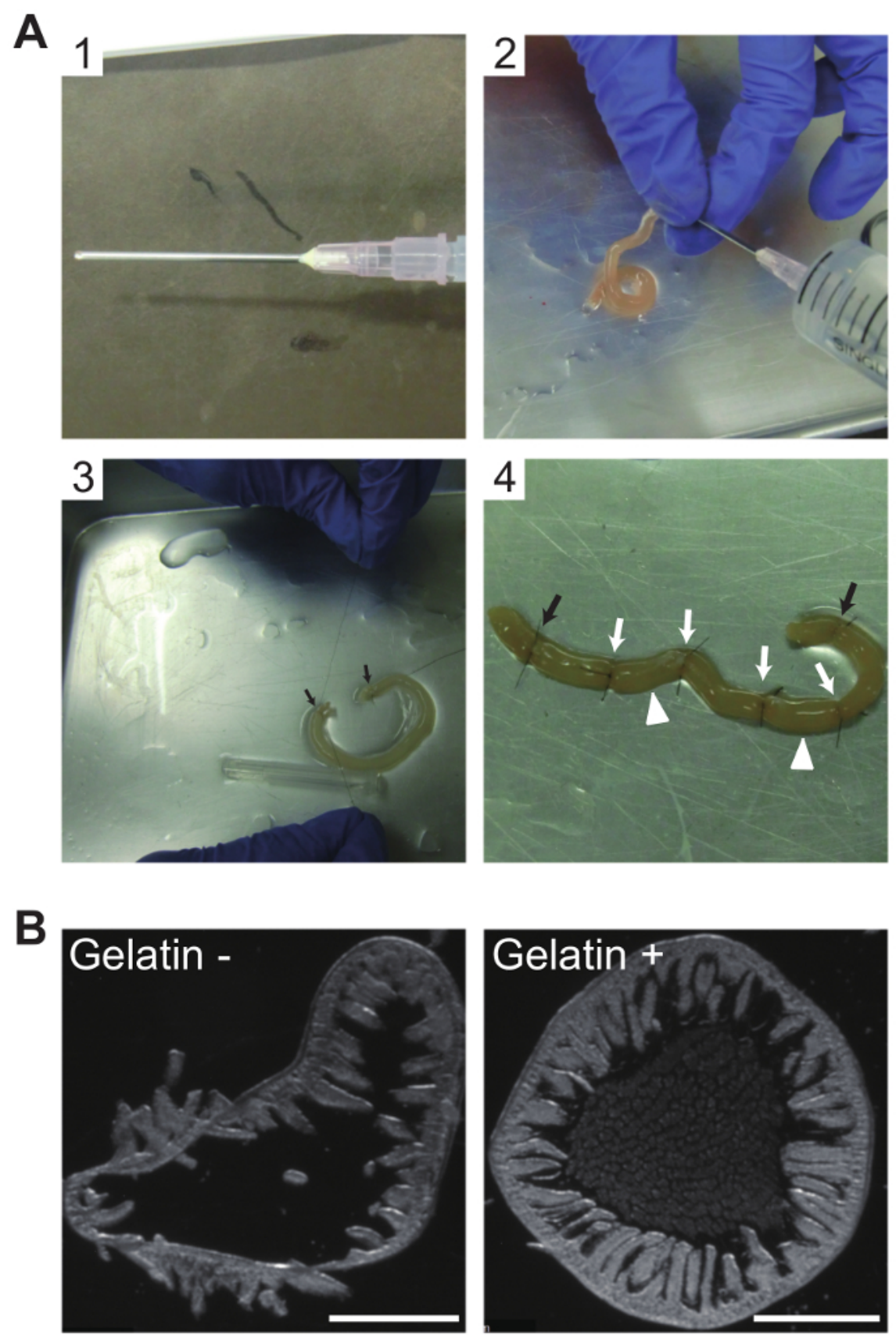

Figure 1: Gelatin filling of jejunum sections for morphological preservation of cryosections

(A) Photographs of procedures for intraluminal filling of mouse jejunum with gelatin. (A1) The beveled tip of an 18-gauge straight needle was removed to avoid piercing the gut wall. (A2) Buffered neutral formalin solution (10\%) was injected in one end of the clipped jejunum to flush the intestinal contents and fix the gut lumen surface. (A3) Clipped jejunum filled with liquid gelatin and both ends ligated using a 6-0 nylon suture (black arrows). (A4) Four more suture knots (white arrows) placed between the pre-existing suture knots (black arrows) yielded three shorter pieces. The tissue will then be separated into three sausage-like pieces at the two indicated positions (white arrowheads). (B) Beneficial effects of gelatin-filling on free-floating cryosection morphology. Without gelatin filling, sections tend to kink, allowing the villi to easily swing backward (left); with gelatin filling, a $30 \mu \mathrm{m}$-thick section maintains a disc shape and the upright position of the villi is preserved (right). Images were photographed using Nomarski differential interference contrast. Scale bars, $1 \mathrm{~mm}$. Please click here to view a larger version of this figure. 
A
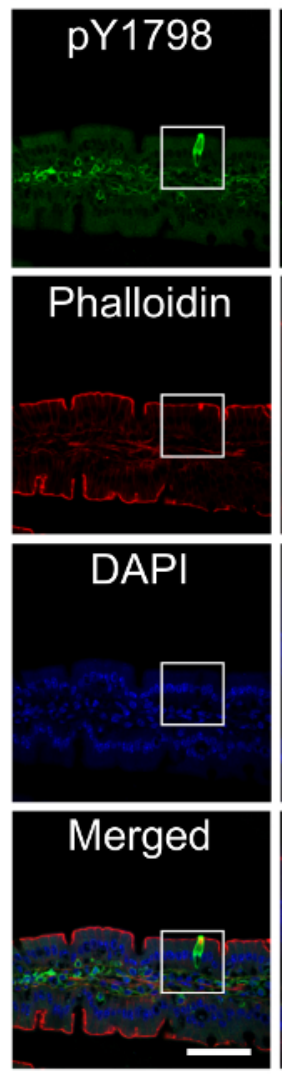
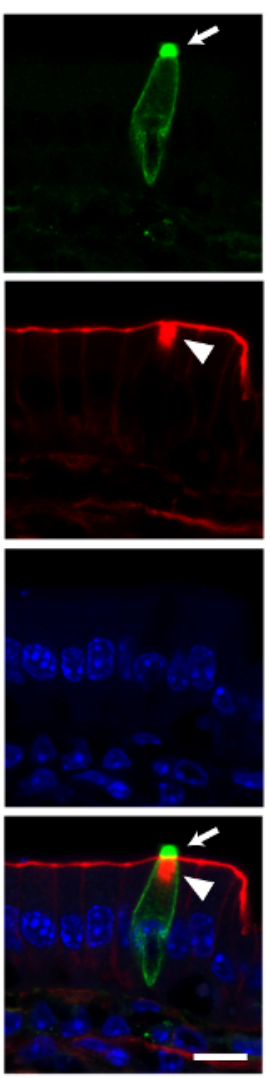

B
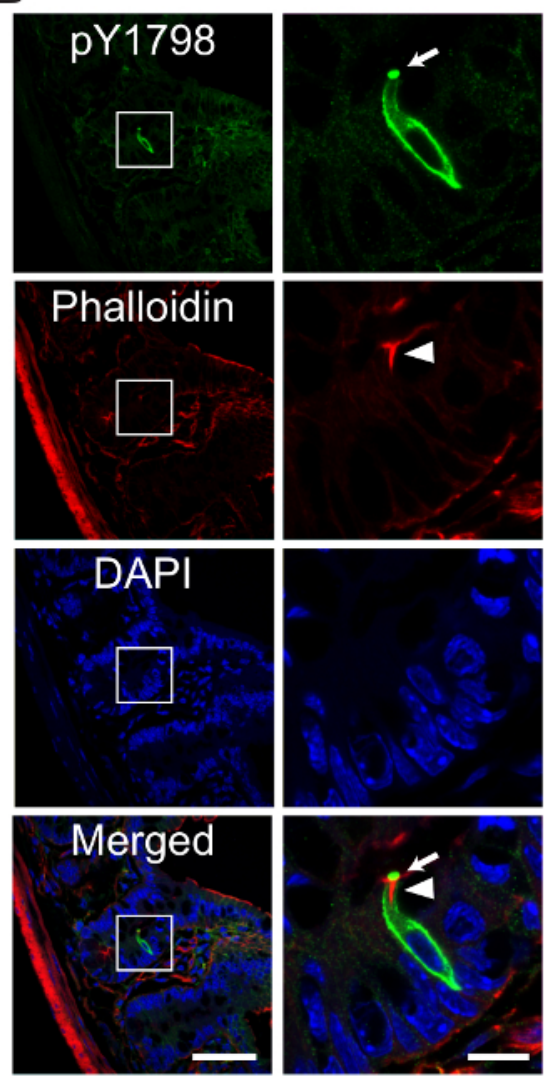

Figure 2: Representative fluorescence images of mouse intestinal tuft cells

Confocal fluorescence images of TCs on a villus (A) or in a crypt (B), in free-floating mouse jejunum sections stained with site-specific and phosphorylation-status-specific antibodies against girdin phosphorylated at tyrosine 1798 (pY1798, green, optimal excitation/emission wavelengths 490/525 nm), phalloidin (red, 590/617 nm), 4,6-diamidino-2-phenylindole (DAPI, blue, 358/461 nm). The area enclosed by white boxes in the low magnification images (scale bars, $50 \mu \mathrm{m}$ ) is expanded on the right (scale bars, $10 \mu \mathrm{m}$ ). pY1798 antibodies reproducibly stain TCs, regardless of location (on a villus (A), or in a crypt (B)), with staining present at the lumenal tip (arrows), membrane, and cytoplasm of the spool-shaped TC soma. A prominently thickened brush border in phalloidin staining (arrowheads) is another distinctive sign of TCs. Please click here to view a larger version of this figure. 


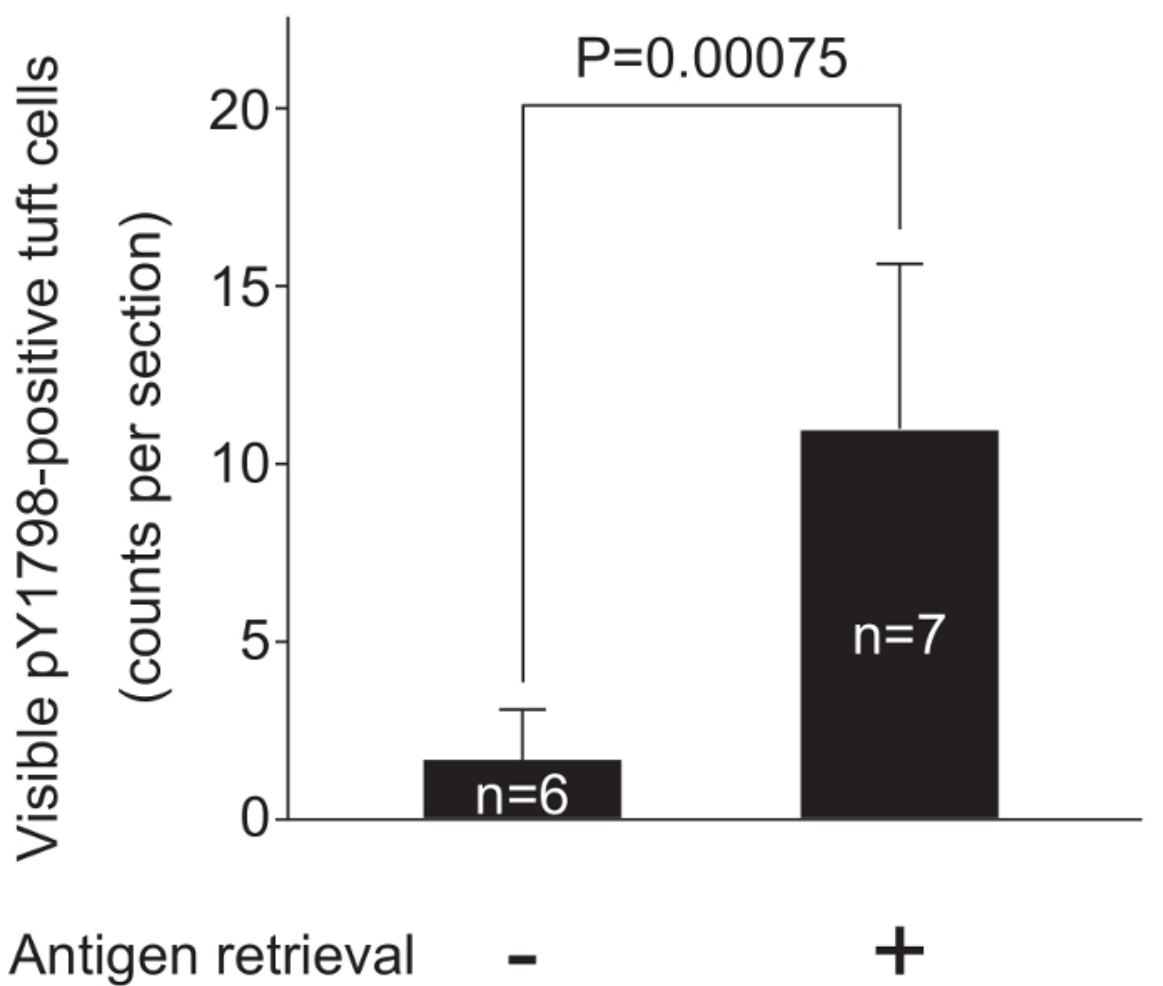

Figure 3: Low-temperature antigen retrieval enhances pY1798 immunofluorescence

Two groups of experimental procedures were compared to confirm the effectiveness of low-temperature antigen retrieval. Free-floating jejunum sections $(30 \mu \mathrm{m}$ thick, $\mathrm{n}=13$ ) from a single frozen block were divided into two groups, and sections from each group were stained following the complete protocol or the same protocol lacking low-temperature antigen retrieval (step 4.2.2). After staining, sections were mounted on individual slides labelled with group numbers, and the labelling on each slide was covered with masking tape. All slides were shuffled, labelled with new numbers on the tape, and observed under fluorescence microscopy through an FITC filter. Total counts of visible pY1798-positive TCs were averaged in each group, and were displayed in a bar graph (mean \pm standard deviation). A two-tailed t-test was performed to compare the average counts between the two groups. P-values below 0.05 were considered statistically significant. Low-temperature antigen retrieval significantly improved the effectiveness of pY1798-immunofluorescence. Please click here to view a larger version of this figure.

\section{Discussion}

This protocol was designed to allow researchers without histology experience to obtain reliable images of tuft cells (TCs). This protocol has three critical points: 1) The use of site- and phosphorylation status-specific rabbit polyclonal antibodies against human girdin phosphorylated at tyrosine 1798 (pY1798 antibodies) that were obtained from a specific company (Immuno-Biological Laboratories = Company X); 2) Gelatin filling of the jejunum; and 3) Low-temperature antigen retrieval. In the broadest sense, phosphorylation status-specific antibodies can be categorized as modification-specific antibodies that recognize a specific type of post-translational modification (e.g. acetylation, methylation, or phosphorylation) of a protein at a specific amino acid residue. Omori et al. and company $\mathrm{X}$ jointly generated pY1798 antibodies by immunizing rabbits with phosphorylated peptide, and purifying the resulting antibodies using solid-phase chromatography with an unphosphorylated peptide following Goto's method ${ }^{9,10}$. pY1798 antibodies were intensively validated with in vitro phosphorylation assays using full-length human girdin expression vectors with/without a point mutation at tyrosine $1798^{9}$. Subsequently, Kuga et al. found that $\mathrm{pY} 1798$ antibodies from company $\mathrm{X}$ are a specific and sensitive marker of intestinal TCs ${ }^{1}$. Thus, inclusion of pY1798 antibodies from company X is a critical point in this protocol.

Gelatin contains collagen extracted from animal tissues and has long been used to embed tissue specimens for histochemical studies using cryosections $^{13}$. Low-melting-point gelatin, which is gelatinized at $4{ }^{\circ} \mathrm{C}$ but melts at room temperature, began to be applied to avoid adverse effects of heat needed to maintain the gelatin in a liquid state during embedding ${ }^{14}$. In this protocol, low-melting-point gelatin made from gelatin powder that gelatinizes at $4{ }^{\circ} \mathrm{C}$ and melts at RT was used to preserve the morphology of transverse cryosections of mouse jejunum. When this gelatin was used to completely fill the jejunum, samples were then formalin-fixed to achieve irreversible gelatinization. Heat-induced antigen retrieval is an effective method to expose antigens that are masked by aldehyde-containing fixatives ${ }^{12}$. However, the high temperatures $(95-99$ ${ }^{\circ} \mathrm{C}$ for $30 \mathrm{~min}$ ) commonly used for analysis of paraffin sections can damage the morphology of the tissue/gelatin complex. Here we used lowtemperature antigen retrieval $\left(50^{\circ} \mathrm{C}\right.$ for $3 \mathrm{~h}$ ) that allows both antigen retrieval and preservation of tissue morphology.

pY1798 antibodies can label TCs not only in mouse jejunum, but also in multiple organs (stomach, ileum, colon, and gallbladder) from mice and humans ${ }^{1}$. However, because pY1798 signals in unfixed tissues from any tissue will rapidly degrade, this protocol includes formalin injection into the jejunum lumen (step 2.1.15., Figure 1A2).

There are limitations associated with the thickness of free-floating cryosections. Although thin cryosections can be advantageous in terms of translucency for microscopy, the thinness makes these sections physically vulnerable. In contrast, thick cryosections are physically sturdy, but have lower antibody permeability into the section. In this protocol, $30 \mu \mathrm{m}$-thick sections were used that were both physically stable and 
permeable to antibodies. Although this method was designed for researchers without extensive experience in histology, if the protocol should fail, pY1798/villin/DAPI triple staining of paraffin sections similar to that used by Kuga et al. could be performed ${ }^{1}$. Paraffin sections were not used here to avoid difficulties associated with phalloidin staining of F-actin in paraffin sections.

Due to the conservation of amino acid sequences adjacent to girdin tyrosine-1798, pY1798 antibodies can be applied to stain TCs in species other than mouse, including rat and human. The gelatin filling used in this protocol can also be applied for other hollow organs. Indeed, apart from pY1798 or TC, the combination of gelatin filling with low temperature antigen retrieval may be useful for revealing notoriously "difficult" epitopes in the mouse gut, and as a consequence, may be of interest for many researchers.

The biological role of girdin and the significance of its phosphorylation in TCs is unclear. However, a study by Lin et al. suggested that tyrosine phosphorylation of girdin is associated with the degree of F-actin polymerization ${ }^{8}$. Meanwhile, Kuga et al. found that lethal doses of apoptosis inducers (e.g., cisplatin, X-ray radiation) caused a large increase in the relative frequency of TCs in the mouse small intestine that they hypothesized may be associated with the possible conversion from enterocytes to TCs, in synchronization with the phosphorylation of girdin in microvilli ${ }^{1}$. This possibility will require additional investigation in the future. Three groups recently observed rapid increase in TC frequency following parasite infection ${ }^{15,16,17}$. Thus, this free-floating staining could be used not only to analyze endoscopically-collected human gut tissues, but TC accumulation could also aid in diagnosis of parasite infection in the human gut.

\section{Disclosures}

The authors have nothing to disclose.

\section{Acknowledgements}

We thank Naoya Asai at Nagoya University for providing useful suggestions for the development of low-temperature antigen retrieval for freefloating jejunum sections. This work was supported by Grants-in-Aid for Scientific Research (KAKENHI) (C) in 2012 (JP25460493) and (B) in 2017 (JP17H04065) from the Japan Society for the Promotion of Science (JSPS), A-STEP grants in 2014 (AS251Z02522Q) and in 2015 (AS262Z00715Q) from the Japan Science and Technology Agency (JST), and a Takeda Visionary Research Grant 2014 from the Takeda Science Foundation (to M.A.).

\section{References}

1. Kuga, D. et al. Tyrosine phosphorylation of an actin-binding protein Girdin specifically marks tuft cells in human and mouse gut. $J$ Histochem Cytochem. 65 (6), 347-366 (2017).

2. Jarvi, O., Keyrilainen, $\mathrm{O}$. On the cellular structures of the epithelial invasions in the glandular stomach of mice caused by intramural application of 20-methylcholantren. Acta Pathol Microbiol Scand Suppl. 39 (Suppl 111), 72-73, (1956).

3. Enomoto, A. et al. Akt/PKB regulates actin organization and cell motility via Girdin/APE. Dev Cell. 9 (3), $389-402$ (2005).

4. Asai, M. et al. Similar phenotypes of Girdin germ-line and conditional knockout mice indicate a crucial role for Girdin in the nestin lineage. Biochem Biophys Res Commun. 426 (4), 533-538 (2012).

5. Kitamura, T. et al. Regulation of VEGF-mediated angiogenesis by the Akt/PKB substrate Girdin. Nat Cell Biol. 10 (3), $329-337$ (2008).

6. Wang, Y. et al. Girdin is an intrinsic regulator of neuroblast chain migration in the rostral migratory stream of the postnatal brain. $J$ Neurosci. 31 (22), 8109-8122 (2011).

7. Nahorski, M.S. et al. CCDC88A mutations cause PEHO-like syndrome in humans and mouse. Brain. 139(Pt4), 1036-1044 (2016).

8. Lin, C. et al. Tyrosine Phosphorylation of the G alpha-Interacting Protein GIV Promotes Activation of Phosphoinositide 3-Kinase During Cell Migration. Science signaling. 4 (192), ra64 (2011).

9. Omori, K. et al. Girdin is phosphorylated on tyrosine 1798 when associated with structures required for migration. Biochem Biophys Res Commun. 458 (4), 934-940 (2015).

10. Goto, H., Inagaki, M. Production of a site- and phosphorylation state-specific antibody. Nat Protoc. 2 (10), $2574-2581$ (2007).

11. Wulf, E., Deboben, A., Bautz, F.A., Faulstich, H., Wieland, T. Fluorescent phallotoxin, a tool for the visualization of cellular actin. Proc Natl Acad Sci U S A. 76 (9), 4498-4502, (1979).

12. Jiao, Y. et al. A simple and sensitive antigen retrieval method for free-floating and slide-mounted tissue sections. J Neurosci Methods. 93 (2), 149-162, (1999).

13. Feltkamp-Vroom, T.M., Boode, J.H. An embedding and sectioning technique for immunohistochemical studies of minute specimens of tissue. J Clin Pathol. 23 (2), 188-189, (1970).

14. Ushida, K. Pre-embedding technique with low melting temperature gelatin for preparation of histological sections. Proceeding of the Annual Meeting on Technologies in Biological Research. 36, 66-69, (2014).

15. Howitt, M.R. et al. Tuft cells, taste-chemosensory cells, orchestrate parasite type 2 immunity in the gut. Science. 351 (6279), 1329-1333 (2016).

16. von Moltke, J., Ji, M., Liang, H.E., Locksley, R.M. Tuft-cell-derived IL-25 regulates an intestinal ILC2-epithelial response circuit. Nature. 529 (7585), 221-225 (2016).

17. Gerbe, F. et al. Intestinal epithelial tuft cells initiate type 2 mucosal immunity to helminth parasites. Nature. 529 (7585), 226-230 (2016) 